\title{
Odontología Forense II: La Identificación Inequívoca
}

\author{
Forensic Dentistry II: The Positive Identification
}

\author{
Gabriel M. Fonseca*; Mario Cantín ${ }^{*+\ldots+*}$ \& Joaquín Lucena
}

\begin{abstract}
FONSECA, G. M.; CANTíN, M. \& LUCENA, J. Odontología Forense II: La Identificación Inequívoca. Int. J. Odontostomat., 7(2):327-334, 2013.
\end{abstract}

RESUMEN: La identificación de cadáveres con importante deterioro debe poseer validez científica, fiabilidad y aplicabilidad en un plazo razonable de tiempo. Entre los métodos primarios, los análisis comparativos odontológicos son apropiados para este fin cuando puede contarse con dos informaciones cotejables: la proveniente de fichas y registros previos de la supuesta víctima -información antemortem (AM)-, y la obtenida de los restos -información postmortem (PM). Tras esta comparación, el odontólogo forense podrá concluir en una identificación inequívoca cuando existe una certeza absoluta de que los datos PM y AM pertenecen a una misma persona. Este procedimiento depende no solo de la resistencia de los tejidos dentarios o sus caracteres individuales, sino también de la disponibilidad, calidad y adecuado procesamiento de toda la información AM y PM disponible. Los errores críticos, aquellos que pueden llevar a una identificación errónea o una falsa exclusión, pueden ser resultado de la ausencia de estándares, la falta de competencia de los profesionales actuantes y el ocultamiento o el fraude. Todas estas situaciones han sido tan reportadas históricamente tanto como los éxitos, aunque escasamente divulgadas en la literatura de habla hispana. Dado que aún en las mejores planificaciones, cada incidente negativo supone un gran desafío de resolución y experiencia, se presenta una revisión de casos y tópicos inherentes a la identificación odontológica, sus necesidades, postulados, limitaciones y nuevos paradigmas, con el objeto de optimizar la flexibilidad y la coordinación necesarias para promover un odontólogo forense no solo idóneo sino también moderado y observador, cualidades necesarias del perito.

PALABRAS CLAVE: odontología forense, identificación inequívoca, identificación errónea, ficha dental.

\section{INTRODUCCIÓN}

La identificación de cadáveres con importante deterioro debe poseer validez científica, fiabilidad y aplicabilidad en un plazo razonable de tiempo. Entre los métodos primarios, y dada la resistencia de las estructuras dentarias, los análisis comparativos odontológicos son apropiados para este fin cuando puede contarse con dos informaciones cotejables: la proveniente de fichas y registros previos de la supuesta víctima -información antemortem (AM)-, y la obtenida de los restos -información postmortem (PM)-. Tras esta comparación, el odontólogo forense podrá concluir en una identificación inequívoca solo cuando existe una certeza absoluta de que los datos PM y AM pertenecen a una misma persona (INTERPOL, 2009). De ser encontradas discrepancias, estas deberán ser explicables por el avance de los procesos biológicos (caries, extracciones, etc.) o la misma intervención de la causa del fallecimiento; de no poder ser explicadas, el resultado del proceso será la exclusión pues se supondrá que ambas informaciones pertenecen a personas diferentes (Varkkola, 2011).

Este procedimiento en apariencia sencillo, depende no solo de la resistencia de los tejidos dentarios o sus caracteres individuales, sino también de la disponibilidad, calidad y adecuado procesamiento de toda la información AM y PM disponible (Brown, 1984). Los "errores críticos", aquellos que pueden llevar a una

\footnotetext{
Profesor Titular, Cátedra de Anatomía Patológica B, Facultad de Odontología, Universidad Nacional de Córdoba, Córdoba, Argentina.

* Programa de Doctorado en Ciencias Morfológicas. CIMA, Departamento Odontología Integral Adultos, Facultad de Odontología, Universidad de La Frontera, Temuco, Chile.

*** Centro de Investigación en Ciencias Biomédicas, Universidad Autónoma de Chile, Temuco, Chile.

**** Profesor Asociado, Departamento de Medicina Forense, Universidad de Cádiz, Cádiz, España.

****** Servicio de Patología Forense, Instituto de Medicina Legal, Sevilla, España.
} 
identificación errónea o una falsa exclusión (Varkkola), pueden ser resultado de la ausencia de estándares, la falta de competencia de los profesionales actuantes y el ocultamiento o el fraude. Todas estas situaciones han sido tan reportadas históricamente tanto como los éxitos, aunque escasamente divulgadas en la literatura de habla hispana. Desde las equívocas consideraciones identificatorias realizadas por el médico español Pablo de Valencia y Forns sobre el cadáver del héroe cubano José Martí en 1907 (Cobo-Abreu et al., 1995), o la fallida usurpación de identidad hecha por Wilhem Beckert en el Crimen de la Legación Alemana, descubierta por el odontólogo chileno Germán Valenzuela Basterrica (Ciocca Gómez, 2010), cada uno de los errores, aciertos y vicisitudes de una identificación dificultosa se presenta como una oportunidad única y fabulosa para aprender, pues a decir de Hinchliffe (2011), ayudan a mejorar las respuestas y la prevención.

Dado que aún en las mejores planificaciones, cada incidente negativo supone un gran desafío de resolución y experiencia, se presenta una revisión de casos y tópicos inherentes a la identificación odontológica, sus necesidades, postulados, limitaciones y nuevos paradigmas con el objeto de optimizar la flexibilidad y la coordinación necesarias para promover un odontólogo forense no solo idóneo sino también moderado y observador, cualidades necesarias del perito.

\section{FICHA, ODONTOGRAMA Y CUANTIFICACIÓN METODOLÓGICA}

La ficha o historia clínica odontológica es la herramienta que utiliza el odontólogo para el registro de la información dental (Ciocca Gómez), y el odontograma o dentigrama, la norma esquemática que le permite la codificación de esa información, no solo en la práctica clínica sino también en la confección de fichas y sistemas específicos para la identificación forense (INTERPOL). Sin embargo, este recurso gráfico aún no cuenta con un consenso de lenguaje internacional y ha sido cuestionada su configuración por la imposibilidad de registrar datos potencialmente identificatorios (Fonseca et al., 2010; Varkkola). Se ha sugerido un mayor recaudo en la obtención y archivo de datos imagenológicos (radiografías, fotografías, modelos, etc) pues permiten registrar información despojada de subjetividad y difícilmente codificable en un odontograma (malposiciones, pigmentaciones, lesiones, etc). Esto posibilita asentar datos morfológicos incluso en ausencia de restauraciones (situaci;on fre- cuente en niños y jóvenes) o edentulismo, y facilita la transmisión de información desligada de barreras lingüísticas (Solheim et al., 1992; Andersen et al., 1995).

Quizás una de las mayores utilidades de codificar la información dental es la posibilidad de cuantificar los rasgos de cotejo y las observaciones. Aunque se ha coincidido en que la comparación de las informaciones AM-PM solo puede ofrecer resultados categóricos: identificación inequívoca, probable, posible, descartada o comparación imposible (INTERPOL), y que la utilización de este modelo cualitativo no implica falta de fiabilidad dada la complejidad de informaciones que deben compararse (Dorion, 1990; Martin de las Heras et al., 2010; Fonseca et al.) la cuantificación analítica del cotejo (tendencia acentuada con la entrada en escena de las pruebas genéticas) exige hoy significación estadística en las metodologías utilizadas. Steadman et al. (2007) en un interesante intercambio epistolar defienden que las comparaciones odontológicas y dactiloscópicas son útiles pero no dan certeza probabilística, pues el resultado categórico de un cotejo de informaciones AM-PM no puede ser corroborado de ninguna manera (Anderson, 2007). Los modelos matemáticos desarrollados en 1977 por Keiser-Nielsen y periódicamente reformulados para poblaciones específicas, continúan defendiendo a las trillonésimas posibles combinaciones de rasgos visibles en las 5 caras de los 32 dientes humanos (rangos similares a los de una perfilación genética) como herramienta de certeza para una identificación inequívoca (Martin de las Heras et al.). Sin embargo, los hechos han logrado rebatir este modelo teórico: ¿cuántas posibilidades hay de que dos personas tengan el mismo odontograma?

Un avión de combate es derribado en Libia. En la noche del 14 de abril de 1986, el F-111 tripulado por los Capitanes de la Fuerza Aérea Norteamericana Fernando Ribas-Dominicci (piloto) y Paul Lorence (copiloto), es derribado en la costa de Libia. El gobierno de ese país recuperó solo un cuerpo poco tiempo después del ataque. Aun cuando su identificación se tornó problemática dada su avanzada descomposición, y que no se utilizaron protocolos específicos, el informe libio de la exploración dental consignó que pertenecía al copiloto. Tres años después, y gracias a la intervención del Vaticano, el gobierno de Libia entregó los restos a la policía italiana. El equipo forense del Instituto di Medicina Legale e delle Assicurazioni en Roma y un experto norteamericano recabaron nueva información PM sobre los restos para cotejar con las 
fichas dentales AM solicitadas a la Fuerza Aérea. Ambos odontogramas AM eran idénticos y coincidían perfectamente con el confeccionado PM (Fig.1). Solo las radiografías $\mathrm{AM}$ permitieron evaluar las formas de las restauraciones, lo que determinó que los restos pertenecían a Ribas-Dominicci y no a Lorence como se había informado originalmente (Cecchi et al., 1997).

\section{ERROR U OMISIÓN EN LOS REGISTROS AM - PM}

Las pericias odontológicas de las 65 víctimas del vuelo 3142 de LAPA colisionado e incendiado en Buenos Aires el 31 de agosto de 1999, permitió la identificación de 39 fallecidos en la primera semana. Sin embargo, este promisorio comienzo se vio luego afectado severamente; las exclusiones logradas por los análisis genéticos en el resto de las víctimas llevaron a sospechar que se habían producido identificaciones cruzadas, por lo que se decidió ampliar la investigación genética a la totalidad de los cuerpos. Catorce víctimas habían sido entregadas a parientes biológicos que no eran tales, nueve de ellas identificadas por comparaciones odontológicas. La causa: solo el $24 \%$ de las fichas odontológicas AM estaban debidamente actualizadas y en nueve casos adicionales existían deficiencias serias que afectaban la calidad de la información (Eleta et al., 2002). Este dato no es excepcional; Soomer et al. (2001), identificando las víctimas estonas del hundimiento del crucero M/S Estonia en 1994, encontraron sólo un $27 \%$ con fichas AM de calidad; Kieser et al. (2006) reportaron que solo $38 \%$ de las víctimas del Maremoto en Tailandia en el año 2004 poseía registros AM aceptables. La falta de regulación legal o la misma negación de los odontólogos a cooperar enviando sus registros dentales fueron las razones más frecuentes. Varkkola afirma que los odontólogos forenses son responsables de la interpretación de los datos AM y de los exámenes PM, pero hasta la más exacta evidencia PM no será de utilidad si los registros AM son insuficientes.

La confección errónea o incompleta de las fichas AM o PM por personal no cualificado ha sido un reporte frecuente en la literatura. En el accidente del Delta Air en Dallas-Fort Worth el 2 de Agosto de 1985, las fichas dentales PM confeccionadas por médicos forenses -antes de que se organizara el equipo de odontólogos-.debieron ser reexaminadas y confeccionadas nuevamente (Dorion). Las identificaciones de las 191 víctimas de las explosiones el 11 de Marzo de 2004 en Madrid (España) expusieron problemas sustanciales en la recolección de la información AM. Los registros médicos y odontológicos fueron recabados por personal policial y voluntarios sin experiencia ni entrenamiento, lo que emanó en una alta insuficiencia de datos imposibles de categorizar (Prieto et al., 2007).
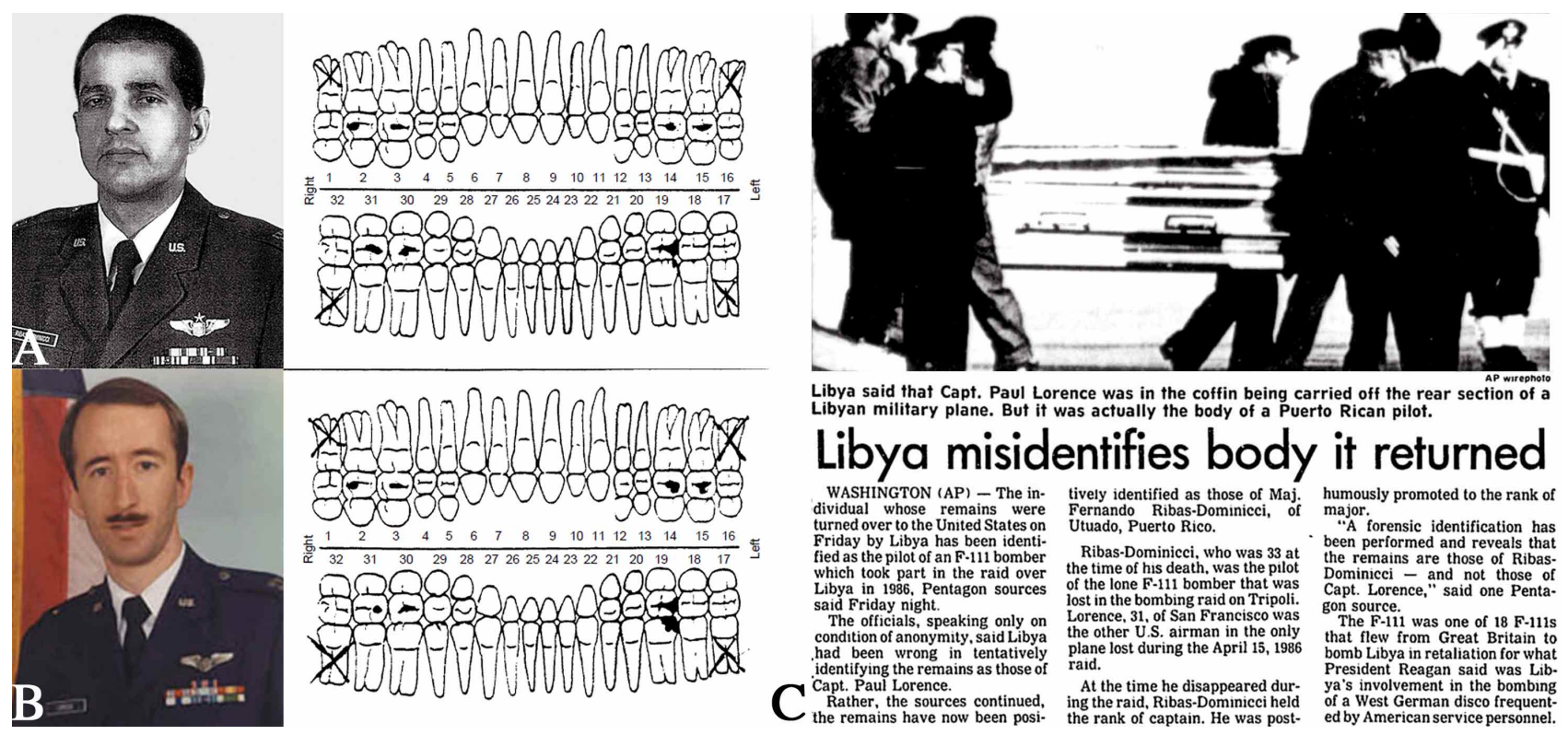

Fig. 1. A. Capitán Fernando Ribas-Dominicci (fotografía Veteran Tributes) y su odontograma AM; B. Capitán Paul Lorence (fotografía San Francisco State University) y su odontograma AM. Nótese la idéntica combinación de patrones en ambos registros de las víctimas (tomados de Cecchi et al., 1997). C. Regreso a los Estados Unidos de los restos de RibasDominicci una vez identificados (The Tuscaloosa News, edición del 14 de Enero de 1989). 
Este problema se ve notablemente incrementado cuando el personal involucrado desconoce terminología, simbología y nomenclatura dental internacional (algo particularmente importante en desastres aéreos). En el accidente del vuelo 759 de Pan Am en New Orleans en 1982, 154 víctimas provenían de 15 países diferentes, lo que significaron nueve tipos distintos de nomenclaturas dentales. Lo que a primera vista aparentaron ser discrepancias resultaron solo en malinterpretaciones (Dorion). Se ha recomendado enfáticamente que un odontólogo forense asista en esta recolección e incluso contacte personalmente (cuando es posible) con el profesional que envía la información, para resolver las explicaciones y abreviaturas utilizadas en las fichas (Solheim et al.).

Uno de los problemas más serios es aquel que involucra al odontólogo que recoge, interpreta y confecciona los registros PM. Ya en 1984, Brown menciona que pueden cometerse errores fácilmente durante este proceso, y que contar con dos odontólogos trabajando juntos, uno examinando y otro registrando (intercambiando sus funciones para control), constituye una medida inteligente para evitar estos sesgos. Esta recomendación ha sido adoptada por INTERPOL en su más reciente guía para la identificación de Víctimas de Catástrofes (IVC) del año 2009. Sin lugar a dudas, las habilidades, experiencia y capacidades del odontólogo serán los elementos clave que podrán afectar de manera directa la calidad de la información a cotejar y el mismo procedimiento de identificación (Hinchliffe).
La Chica del Pijama Amarillo. El 1 de septiembre de 1934, un joven granjero descubre en una zanja en Albury, Australia, el cuerpo quemado de una joven mujer vestida con un pijama amarillo. Tras infructuosos y demorados intentos de identificarla (de hecho, llegaron a exponerse publicamente los restos), la policía convocó al Dr. Francis Herbert Jackson, odontólogo sin experiencia previa forense. Luego de tres días de trabajo, su informe refirió la presencia de 6 dientes restaurados y 7 ausentes $A M$, entre estos últimos, el primer molar superior derecho. En julio de 1935 en Melbourne, la policía entrevistó a un periodista italiano de nombre Antonio Agostini cuya esposa, Linda Platt Agostini, había sido reportada como desaparecida (Fig. 2). Este no reconoció al cadáver pero ofreció información respecto del odontólogo que trataba a su esposa. La información provista por este nuevo profesional resultó incompatible con la PM obtenida por Jackson: Linda Platt Agostini poseía 8 dientes restaurados incluyendo al primer molar superior derecho. No fue sino hasta diez años después, en 1944, cuando una nueva pericia odontológica sobre el cadáver reveló restauraciones estéticas de silicato en cada uno de los segundos premolares, y la presencia del primer molar superior derecho. El segundo molar era el ausente y el tercero había ocupado su lugar en el arco. Esto sí concordaba con los registros AM lo que confirmó al cuerpo como perteneciente a Linda Platt Agostini. Confrontado con esta identificación, Antonio Agostini admitió haber asesinado a su esposa. Jackson debió reconocer en el estrado su equivocación en los primeros re-

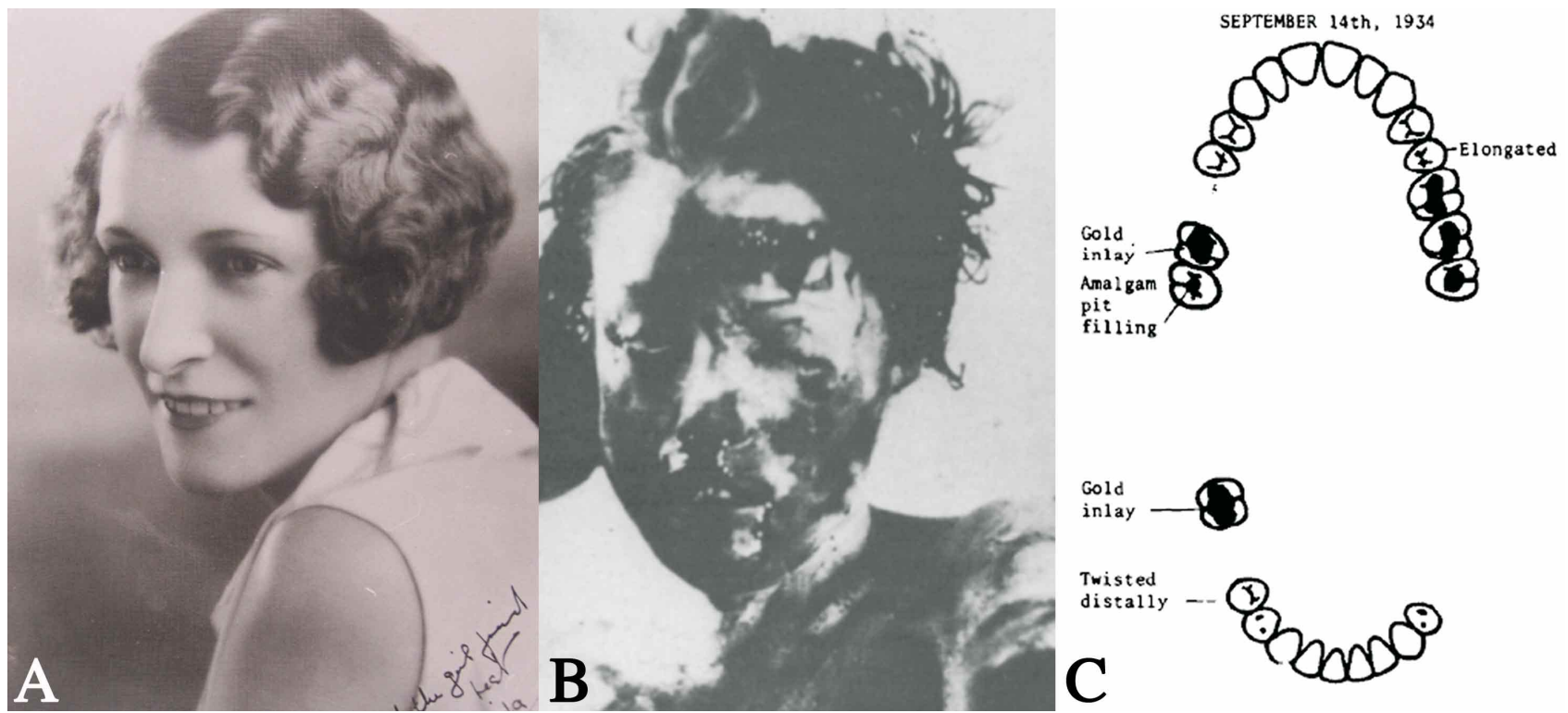

Fig. 2. A. Linda Platt Agostini, 1930 (Fotografía original, archivos de la policía estatal de Nueva Gales del Sur (Australia); B. Imagen de "La Chica del Pijama" (tomado de Brown, 1982); C. Odontograma PM confeccionado por el odontólogo Dr. Francis Herbert Jackson el 14 de Septiembre de 1934, para distribución policial (tomado de Brown, 1982). 
gistros PM, justificándose en su inexperiencia (Brown, 1982). Aun hoy sigue cuestionándose toda la evidencia recogida y la misma identificación de la víctima.

\section{OCULTAMIENTO, USURPACIÓN, SUSTITUCIÓN Y FRAUDE DE IDENTIDAD}

Aunque las diferentes legislaciones insisten en emplear metodologías científicas para la identificación, suele darse por sentada la inexistencia de variables intencionales para distorsionar este procedimiento: la manipulación ex profeso de la información AM promoviendo el ocultamiento, la usurpación o la sustitución de identidad en un contexto delictivo, ha originado no pocas situaciones embarazosas (Fonseca et al.; McLemore et al., 2011).

La Guía IVC presentada por INTERPOL en el año 1997 hace una singular pregunta: “¿Podría haberse puesto un nombre equivocado en la lista de víctimas por error, o fraudulentamente por alguien que tratara de cambiar de identidad?" (Fonseca et al.). Esta no es una pregunta menor, ya en 1990, Dorion recomienda tener presente en accidentes aéreos la posibilidad de sustitución intencional de un cuerpo por otro, lo que invariablemente va a conducir a una identifica- ción falsa. Aunque no es un tópico recurrente en la literatura (de hecho la actual versión 2009 de la Guía IVC de INTERPOL no hace mención alguna a estos supuestos), Krompecher et al. (2000) recomiendan completar absolutamente todos los protocolos de identificación biológica, sobre todo en casos controvertidos; Brown (2007) subraya la importancia de entregar y resguardar las fichas clínicas dentales con sometimiento a estrictas cadenas de custodia; y Cattaneo et al. (2010) proponen fundamentar la identificación exclusivamente en caracteres biológicos, evitando con ello una identificación errónea producto del cambio casual o intencional de objetos o vestimentas supuestamente "personales".

La adulteración de un odontograma. El 23 de Marzo de 1979 , el cuerpo desnudo y atado de una mujer fue encontrado en una hondonada montañosa en Suiza. Si bien se encontraba en descomposición, la preservación de su dentadura permitió confeccionar su ficha PM y hacer una estimación de su edad. El envío de esta información a la prensa y a varios departamentos policiales del país favoreció la llegada de diferentes informes odontológicos de algunas personas denunciadas como desaparecidas. Uno de los odontogramas recibidos resultó particularmente extra-

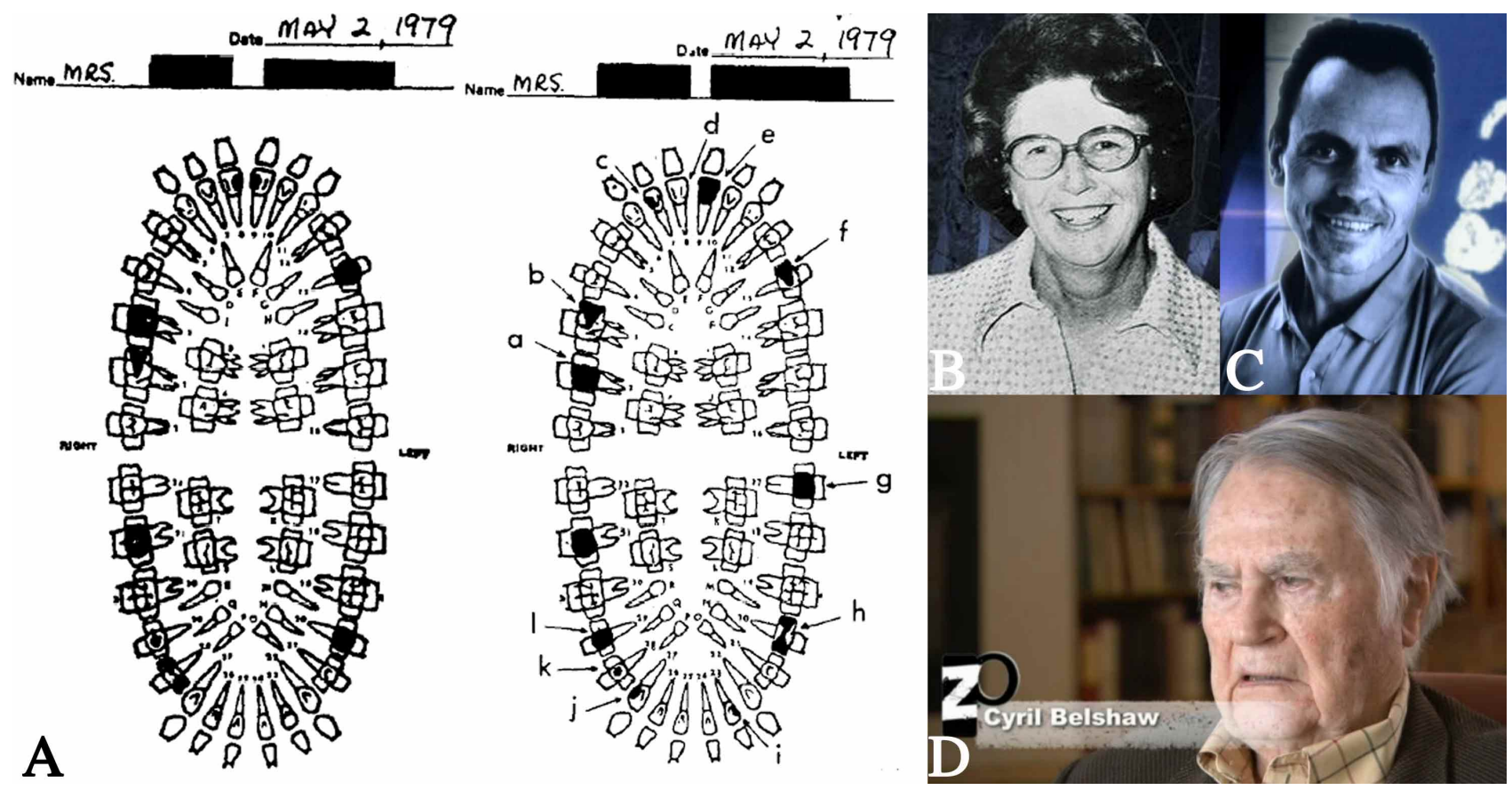

Fig. 3. A. Odontograma original y adulterado. Las flechas muestran las modificaciones realizadas por Cyril Belshaw, marido de la víctima Betty Belshaw (tomado de Imobersteg, 1982); B. Betty Belshaw; C. El odontólogo forense Claude Imobersteg; D. Cyril Belshaw en la actualidad; si bien reconoce la adulteración del odontograma, continúa defendiendo su inocencia respecto del homicidio de su esposa (fotogramas tomados de "De la disparition à la mort de Betty Belshaw" documental transmitido por "Zone d'ombre" para la Cadena RTS Radio Télévision Suisse, 12 de Junio de 2013). 
ño. Si bien constaban el nombre de la paciente y símbolos claramente de uso profesional, no figuraban mayores datos, ni siquiera el nombre del odontólogo tratante. Los rasgos dentales descriptos en el odontograma no coincidían con los de la fallecida. Esta ficha pertenecía a Betty Belshaw, una mujer canadiense desaparecida en París (a unos $500 \mathrm{~km}$ ) tres meses antes, y había sido entregada por su esposo, Cyril Belshaw, ya de retorno en Canadá para esa fecha. La Policía regional solicitó a la Real Policía Montada Canadiense (RCMP) la búsqueda de mayores datos sobre la desaparecida, en particular de su odontólogo y de los tratamientos dentales realizados. Al día siguiente de haber sido contactado el profesional y luego de haber sido informado de este suceso, el esposo de Betty envió una carta a la RCMP admitiendo haber adulterado el odontograma enviado a la policía suiza. Con la nueva información y la ficha original, el cadáver fue finalmente identificado como perteneciente a la mujer canadiense. Una vez arrestado, el esposo confesó haber fotocopiado el odontograma inicial -el que solicitó en su momento al odontólogo tratante- , borró algunos rasgos del mismo y luego con bolígrafo rediseñó los patrones dentales a su necesidad. La ficha recibida por la policía suiza era una nueva fotocopia del documento ya adulterado. Cyril Belshaw se desempeñaba como Profesor Titular del Departamento de Antropología en la Universidad de Vancouver (Canadá) (Imobersteg, 1982). Luego de un controvertido juicio, Belshaw fue liberado por el beneficio de la duda. El crimen permanece en la actualidad sin resolver (Fig. 3).

\section{DISCUSIÓN}

Para Page et al. (2011) no existe argumento alguno que logre probar la asunción de unicidad como carácter para la identificación. Aunque los intentos por confirmarla en ciencias forenses han postulado complejas formulaciones matemáticas para calcular probabilidades de coincidencia, tales datos tienen poca significación práctica cuando en la realidad, los análisis forenses no siguen la misma vía. Ningún dispositivo tecnológico o modelo cuantitativo puede reemplazar la realidad percibida por el ojo humano. Ningún sistema informático puede substituir la experiencia práctica de los odontólogos operándolo (Kieser et al.). El hecho de que la dentición humana pueda ser considerada única, aún como concepto limitado a la población finita disponible a los investigadores, se hace irrelevante considerando la tarea que se le solicita al odontólogo (Page et al.).
La pesadilla del odontólogo forense es equivocar una identificación. Posee serias connotaciones legales tanto en lo civil como en lo penal, razón por la que uno no debería permitirse errar una identificación (Dorion). Aunque razones de estas falsas identificaciones pueden ser el no contar con los tiempos apropiados, la inexperiencia o las constantes presiones externas (autoridades, prensa, familiares, etc) (Kieser et al.; Hinchliffe), el mayor e imperdonable error será sin dudas la incompetencia, la no cualificación para llegar a la opinión experta. Dorion afirma que no hay vergüenza alguna en ser meticuloso y prudente, y que intentando "quedar bien", el perito puede arribar a conclusiones de identificaciones inequívocas sin poseer suficiente evidencia que las sustente. Aunque una identificación forense con estándares bajos puede no ser considerada "antiética", la posibilidad de originar una falsa identificación supera por largo a sus ventajas potenciales. De sospecharse una identificación errónea, deberían solicitarse caso por caso nuevos exámenes de los restos y sus correspondientes análisis genéticos para ponerlos de manifiesto (ICRC, 2009). Por desgracia los casos hoy continúan, y las causas siguen siendo las mismas.

Las diferentes experiencias han originado recomendaciones para los procesos de comparación de las informaciones AM-PM: deberán reunirse coincidencias suficientes en cantidad y calidad, y de existir discrepancias, estas deberán poder explicarse lógica o biológicamente; el lapso desde la última intervención odontológica reseñada en la ficha no debería exceder los 3 años en situaciones de discrepancias relativas; los estigmas o intervenciones odontológicas referidos por personas allegadas tendrán solo un valor complementario; ante una mínima duda respecto de los puntos anteriores, la identificación odontológica deberá complementarse con otros métodos identificatorios (Eleta et al.). Kieser et al., subrayan que en casos de víctimas de muchas nacionalidades, la información $A M$ debe ser recolectada en el país de origen por odontólogos entrenados en conocimientos forenses.

El único alivio para los familiares de un desaparecido reside en la confirmación creíble de su muerte y en el conocimiento de que los restos han sido o serán tratados con dignidad, de acuerdo a sus creencias culturales o religiosas (ICRC). Cada caso debería ser cuidadosamente considerado antes de emitir una conclusión de identificación: los errores conducen a un mayor sufrimiento para los familiares del fallecido (Hinchliffe). Ante la pregunta de qué pueden hacer los peritos mientras esperan que la ciencia arroje luz a los particulares 
inconvenientes inherentes a las metodologías empleadas, Saks (2010) responde con mucha claridad: actuar con honestidad y humildad, limitar los informes y pericias a lo probado en campo sin generalizar conclusiones, abandonar los argumentos dogmáticos y la terminología engañosa y ofrecer conclusiones modestas pero precisas, reconociendo que la identificación forense es todavía un esfuerzo probabilístico.

AGRADECIMIENTOS Al Dr. José Luis Prieto Carrero, Médico Forense y Odontólogo, Especialista en
Antropología Forense y Profesor Asociado de la Universidad Complutense de Madrid por la información facilitada de lo acontecido en los atentados de la estación de Atocha (Madrid, España) el 11 de Marzo de 2004.

A la Comisión Nacional de Investigación Científica y Tecnológica de Chile (CONICYT) a través de su Programa de Inserción y Atracción de Capital Humano Avanzado MEC, Proyecto Folio 80120014 CONICYT, Chile, por la ayuda prestada para el desarrollo de este estudio.

FONSECA, G. M.; CANTÍN, M. \& LUCENA, J. Forensic dentistry II: The positive identification. Int. J. Odontostomat., 7(2):327-334, 2013.

ABSTRACT: The identification of destructed human bodies must have scientific validity, reliability and applicability in a reasonable time frame. Between the primary methods, the comparative dental analyses are useful to this aim when two types of data are available to compare: the one obtained from previous records of the supposed victim - the antemortem data (AM)-, and the obtained of the remains - the postmortem data (PM). After this match, the forensic odontologist will be able to conclude in a positive identification when the AM and PM data match in sufficient detail to establish that they are from the same individual. This procedure depends not only on the resistance of the dental tissues or their individual characters, but also on the availability, quality and suitable processing of all the available AM and PM data. The critical errors, which can lead to misidentifications or false exclusions, can be a result of the absence of standards, the lack of professional competencies, hiding or fraud. All these circumstances have been reported historically as well as the successes, but scantily informed in the Spanish literature. Since that, still in the best planning, every negative incident supposes a great challenge of resolution and experience. In this paper we present a review on cases and topics inherent to the dental identification, its needs, postulates, limitations and new paradigms to optimize the necessary flexibility and coordination to promote a forensic odontologist not only competent but also moderate and alert, necessary qualities of the expert witness.

KEY WORDS: forensic dentistry, positive identification, misidentification, dental chart.

\section{REFERENCIAS BIBLIOGRÁFICAS}

Andersen, L.; Juhl, M.; Solheim, T. \& Borrman, H. Odontological identification of fire victims - potentialities and limitations. Int. J. Legal Med., 107(5):229-34, 1995.

Anderson, B. E. Statistical basis for positive identification in forensic anthropology. Am. J. Phys. Anthropol., 133(1):741; author reply 741-2, 2007.

Brown, K. A. Dental Identification of Unknown Bodies. Ann. Acad. Med. Singapore, 13(1):3-7, 1984.

Brown, K. A. The identification of Linda Agostini. The significance of dental evidence in the Albury 'pyjama girl' case. A case report. Forensic Sci. Int., 20(1):81-6, 1982.

Brown, K. A. Procedures for the collection of dental records for person identification. J. Forensic Odontostomatol., 25(2):63-4, 2007.

Cattaneo, C.; Porta, D.; De Angelis, D.; Gibelli, D.; Poppa, P.
\& Grandi, M. Unidentified bodies and human remains: An Italian glimpse through a European problem. Forensic Sci. Int., 195(1-3):167.e1-6, 2010.

Cecchi, R.; Cipolloni, L. \& Nobile, M. Incorrect identification of a military pilot with international implications. Int. J. Legal Med., 110(3):167-9, 1997.

Ciocca Gómez, L. Odontología Médico-Legal. Aspectos Forenses, Profesionales y Sociales. Santiago, Ed. Jurídicas de Santiago, 2010.

Cobo-Abreu, A. R.; González-Pérez, J. \& Cobo-Montañés, Y. Reseña histórica: identificación de los restos mortales de José Martí, mediante el aparato dental. Med. Leg. Costa Rica, 11-12(2-1):46-9, 1995.

Dorion, R. B. Disasters big and small. J. Can. Dent. Assoc., 56(7):593-8, 1990. 
Eleta, G.; Odzak, J.; Bosio, L. \& Sotelo Lago, R. Identificación en desastres de masas. Cuad. Med. Forense, 1(3):167-87, 2002.

Fonseca, G. M.; Viganó, P. \& Olmos, A. Odontoidentificación, "Falsas apariencias" y "Los Cazadores de Mitos". Cuad. Med. Forense, 16(4):205-15, 2010.

Hinchliffe, J. Forensic odontology, part 2. Major disasters. Br. Dent. J., 210(6):269-74, 2011.

ICRC - International Committee of the Red Cross. Missing people, DNA analysis and identification of human remains. $2^{\circ} \mathrm{Ed}$. Geneva, ICRC, 2009

Imobersteg, C. The falsification of an odontogram. Forensic Sci. Int., 20(1):77-9, 1982.

INTERPOL. Guía para la Identificación de Víctimas de Catástrofes. 2009. Disponible en: http://www.interpol.int/ content/download/10461/74528/version/4/file/ guide[1].pdf

Kieser, J. A.; Laing, W. \& Herbison, P. Lessons learned from large-scale comparative dental analysis following the South Asian tsunami of 2004. J. Forensic Sci., 51(1):10912, 2006.

Krompecher, T.; Brandt-Casadevall, C.; Horisberger, B.; Perrier, M. \& Zollinger, U. The challenge of identification following the tragedy of the Solar Temple (Cheiry/Salvan, Switzerland). Forensic Sci. Int., 110(3):215-26, 2000.

Martin-de-Las-Heras, S.; Valenzuela, A.; Luna, Jde D. \& Bravo, M. The utility of dental patterns in forensic dentistry. Forensic Sci. Int., 195(1-3):166.e1-5, 2010.

McLemore, J.; Hodges, W. \& Wyman, A. Impact of identity theft on methods of identification. Am. J. Forensic Med. Pathol., 32(2):143-5, 2011.

Page, M.; Taylor, J. \& Blenkin, M. Uniqueness in the forensic identification sciences--fact or fiction? Forensic Sci. Int., 206(1-3):12-8, 2011.

Prieto, J. L.; Tortosa, C.; Bedate, A.; Segura, L.; Abenza, J. M.; Mariscal de Gante, M. C.; et al. The 11 March 2004 Madrid terrorist attacks: the importance of the mortuary organisation for identification of victims. A critical review. Int. J. Legal Med., 121(6):517-22, 2007.

Saks, M. J. Forensic identification: From a faith-based "Science" to a scientific science. Forensic Sci. Int., 201(13):14-7, 2010.

Solheim, T.; Lorentsen, M.; Sundnes, P. K.; Bang, G. \& Bremnes, L. The "Scandinavian Star" ferry disaster 1990-a challenge to forensic odontology. Int. J. Legal Med., 104(6):339-45, 1992.
Soomer, H.; Ranta, H. \& Penttilä, A. Identification of victims from the M/S Estonia. Int. J. Legal Med., 114(4-5):25962, 2001.

Varkkola, O. Dental identifications and aspects of medicolegal investigations of the Finnis Victims of the SumatraAndaman Earthquake on 26 December 2004. Academic Dissertation, Hjelt Institute, Department of Forensic Medicine, University of Helsinki, Finland, 2011.

Dirección para Correspondencia:

Gabriel M. Fonseca, D.D.S., Ph.D.

Mariano Moreno 937, Cordoba

Zip code: X5000MRS.

ARGENTINA

Email: gabriel_fonseca@argentina.com

Recibido : 06-03-2013

Aceptado: 11-04-2013 